\title{
Depression in Osteoarthritis: Current Understanding
}

\author{
Shen-Tao Wang (D), Guo-Xin $\mathrm{Ni}$ (D) \\ School of Sport Medicine and Rehabilitation, Beijing Sport University, Beijing, People's Republic of China \\ Correspondence: Guo-Xin Ni, Tel +86-10-62989780, Fax +86-10-62989670, Email niguoxin@bsu.edu.cn
}

\begin{abstract}
Depression, one of the most common comorbidities with osteoarthritis (OA), affects patient prognosis and quality of life. It also increases the overall burden of disease. This subgroup of patients has not been effectively managed in clinical settings. The study aimed to direct physicians' attention to the co-occurrence of depression and OA. Therefore, this review summarizes the relevant literature published over the past 10 years. The focus is on the prevalence of and risk factors for depression in OA, the effects of depression on OA development and treatment response, comorbidity mechanisms, screening, and non-pharmacological treatment. The research on the etiology of depression has been driven largely by epidemiological studies. Recent studies have shown that high levels of pain, poor levels of function, high numbers of OA sites, and slow gait might be associated with depression. However, the pathophysiology of OA and depression comorbidities remains unclear. In addition to immune inflammation and structural changes in the brain, which have been documented in brain imaging studies, psychosocial factors may also play a role. The evidence indicates that depression can be treated with early intervention; however, adjustments may need to be made for individuals with comorbid depression in OA. It is recommended that health care providers pay more attention to depressive symptoms in patients with OA. Clinicians should develop and implement an individualized and comprehensive treatment plan for patients based on a mental health assessment and in teams with other professionals to optimize treatment outcomes.
\end{abstract}

Keywords: osteoarthritis, depression, epidemiology, prognosis, screening, treatment

\section{Introduction}

Osteoarthritis (OA), a chronic joint disease characterized by pain and limited mobility, affects over 300 million people worldwide. ${ }^{1}$ According to the disease cost calculation method of Puig-Junoy et al, the social cost of OA may be $0.25 \%$ to $0.50 \%$ of a country's Gross Domestic Product. ${ }^{2}$ There is consensus around the concern that the overall burden of OA will increase as the population ages. ${ }^{3}$

Depression is common in people with OA, with a prevalence of $20 \% .{ }^{4}$ In 2020 , a multicenter cross-sectional study conducted in Australia and the United States that included 19,110 participants found that compared to the non-OA group, the Odds ratio (OR) for depressive symptoms in the OA group was $1.41\left[(95 \%\right.$ confidence interval $(\mathrm{CI})=1.27-1.57) .{ }^{5}$ The reported prevalence of depression among patients treated with total shoulder arthroplasty increased from $5.1 \%$ in 2002 to $15.4 \%$ in $2012 .{ }^{6}$ This represents an almost three-fold increase. A similar trend has been observed among patients with total knee arthroplasty (TKA). ${ }^{7}$ Considering the large population of patients with OA, a small percentage translates into a significant number of people. Of concern is the accumulating evidence that depression negatively affects prognosis, ${ }^{7}$ healthcare expenditures, ${ }^{8}$ and quality of life (QoL). ${ }^{9}$ Specifically, individuals with both OA and depression have $38.8 \%$ higher direct medical costs than those with only OA. ${ }^{8}$ Data from the 2014 Japanese National Health and Wellness Survey showed significant differences in Health-related quality of life (HRQoL), work productivity barriers, and health care resource utilization between those with and without moderate to severe depression, even after adjusting for potential confounders. ${ }^{9}$ The public health implications of these findings are vast, as it is suggested that depression exacerbates the health-related burden of OA and that more attention should be paid to depression in people with OA. However, depression is often neglected. In particular, identification and diagnosis of depression is more difficult when 
prominent somatic symptoms of OA obscure less obvious depressive symptoms. ${ }^{8,10}$ These sobering and salient evidence emphasize the pressing need for a deeper understanding of this relationship.

Since a large number of studies has investigated the bidirectional relationship between OA and depression over the years, we have seen the need for a more up-to-date review. This narrative review aimed to (1) summarize the relevant epidemiologic literature; (2) explore the effects of depression on the onset, progression, and prognosis of OA and determine the mechanisms of OA with comorbid depression; and (3) discuss the extant knowledge on depression screening and nonpharmacological treatments in OA.

\section{Depression and OA: Epidemiological Association}

At the broadest level, a large and still growing literature documents the association of OA with depression. According to the National Health Interview Survey (2015-2017), the age-standardized prevalence of depressive symptoms in adults with arthritis was $12.1 \%$; however, in adults without arthritis, it was $4.7 \% .{ }^{11}$ In addition, the prevalence of depression in specific forms of arthritis has been investigated. In 2016, a systematic review found a pooled prevalence of depressive symptoms among patients with OA of $19.9 \%$ (95\% CI: $15.9-24.5 \%){ }^{4}$ Furthermore, the prevalence of depressive symptoms among adult knee osteoarthritis (KOA) patients and mixed lower extremity OA patients was $18.5 \%(95 \%$ CI: $13.8-23 . \%)$ and $23.0 \%$ (95\% CI: $16.4-30.2 \%)$, respectively. ${ }^{4}$ A meta-analysis by Scott et al found that approximately one in five total joint arthroplasty (TJA) patients had depressive symptoms before and three months after surgery, and this figure remained at $13 \%$ at one year after surgery. ${ }^{12}$ Historically, OA has received significantly less research attention compared to rheumatoid arthritis (RA). After a long period of neglect, the number of studies documenting the prevalence of depression in OA has increased in the past decade. Studies in the United States, ${ }^{8}$ Canada, ${ }^{13}$ Australia, ${ }^{5}$ Germany, ${ }^{14}$ Portugal, ${ }^{15}$ China, ${ }^{16}$ Japan, ${ }^{9}$ and Korea ${ }^{17}$ have provided evidence of an association between OA and depression.

\section{OA as a Risk Factor for Depression}

Depression is known to be a complex multifactorial disorder. In the OA population, the depression risk factors involved can usually be divided into general factors and factors related to disease severity. Early studies were mostly cross-sectional studies conducted in different settings, and researchers wanted to understand the prevalence of major depressive disease (MDD) and depressive symptoms in the OA population, and to analyze the differences in characteristics between people with OA with and without depressive symptoms. In the German primary care population, almost $20 \%$ of OA patients have been found to have moderately severe depression. ${ }^{14}$ Moreover, the mean scores of the Patient Health Questionnaire-9 (PHQ-9) were quite high, with a mean of $>15$. Pain, social contact, physical limitations and Body Mass Index (BMI) were modifiable risk factors associated with the severity of depression, while increasing age was a lower predictor of PHQ-9 scores. ${ }^{14}$ Similarly, Sale and Hawker et al found a $21 \%$ prevalence of depression assessed using the Center for Epidemiological Studies-Depression scale (CES-D) in a cohort of 1227 OA patients. ${ }^{18}$ Being female, experiencing greater pain and fatigue, experiencing stressful life events, being treated for depression/psychiatric disorders, and higher levels of coping behaviors were associated with depression. ${ }^{18}$ Another cross-sectional study showed a $12.2 \%$ prevalence of depression among patients with end-stage hip osteoarthritis (HOA) and KOA. ${ }^{13}$ Patients reporting depression were more likely to be female, have a higher BMI, a higher number of comorbidities and a higher number of painful joints. ${ }^{13}$

In contrast to cross-sectional studies that focus on providing prevalence data, longitudinal studies can better show the temporal order between variables as well as identify potential factors that contribute to depression in people with OA. Veronese et al showed that individuals with multiple OA sites and those with lower extremity OA were more likely to develop depressive symptoms than the cohort without OA. ${ }^{19}$ Unadjusted analyses showed that those with OA had increased odds of depressive symptoms at four years of follow-up compared to those without OA. Notably, after adjusting for 12 potential confounders, having OA at baseline did not significantly increase the odds of depressive symptoms compared to those without $\mathrm{OA}(\mathrm{OR}=1.26$; $95 \% \mathrm{CI}: 0.95-1.67, \mathrm{P}=0.25) .{ }^{19}$ The significant association in univariate analysis became nonsignificant after adjusting for variables, possibly indicating that the association between OA and depression onset was confounded by other factors. A recent longitudinal study of approximately 500,000 Swedish residents investigated the temporality between OA and comorbidity, similarly finding that those with OA had an increased risk of being subsequently diagnosed with depression compared to those without OA. ${ }^{20}$ Another longitudinal 
study also found that female, low income and smoking were predictors of rapidly worsening depressive symptoms in KOA patients. ${ }^{21}$ Besides focusing on radiographic OA or physician-diagnosed OA, Sugai et al observed the impact of knee pain and dysfunction on the development of depression in a community-based 2-year cohort and showed that older adults with severe pain (pain at night while in bed) and dysfunction (difficulty putting on socks, getting into and out of a car, taking off socks) were at significantly increased risk of developing depressive symptoms. ${ }^{22}$ Therefore, the authors concluded that when going outside begins to become a physical burden for older adults, they are likely to develop depression. ${ }^{22}$ This view was subsequently supported by White et al, using data from the Osteoarthritis Initiative, who found that slow gait speed (20-meter walk $<1.2 \mathrm{~m} / \mathrm{s}$ ) was a risk factor for worsening depressive symptoms in individuals with or at high risk for KOA. ${ }^{23}$ Recently, Zheng et al found that patients who had KOA and two or more painful body sites at baseline were at increased risk for depression. ${ }^{16}$

But not all studies had consistent findings. Using the Osteoarthritis Initiative data, Rathbun et al found that increased structural severity and decreased physical performance in patients with radiological KOA increased the risk of depression onset. ${ }^{24}$ However, after adjusting for time-varying confounders, pain severity was not significantly associated with the onset of depression. ${ }^{24}$ This seems to be an important finding, or at least one worth pondering. Regrettably, the authors did not provide a more in-depth analysis of this. In our opinion, this does not mean that OA pain has no effect on depressive symptoms. This variation in the depression-knee pain correlation may depend on the specific study design, sample size, sample inclusion criteria, sample characteristics, and statistical modeling methods. Actually, as a direct stressor, pain has been demonstrated as a risk factor for depression. ${ }^{25} \mathrm{~A}$ recent meta-analysis including 121 studies [24 randomized controlled trials (RCTs), 36 cross-sectional studies and 61 cohort studies] further supports a moderate positive correlation between OA pain severity and depression severity in patients with OA $(\mathrm{r}=0.36, \mathrm{p}<0.001) .{ }^{26}$ Indeed, these studies suggest that OA symptom burden, particularly pain and physical function limitations, play a prominent role in the development of depression.

Sayre et al conducted a cohort study designed to identify risk factors for future depression in KOA. ${ }^{27}$ Interestingly, the authors found that compared to non-OA, Kellgren-Lawrence grade (KL) 1 levels were predictive of depression in the present and at follow-up, but KL 2 and KL 3 levels were not predictive. ${ }^{27}$ A seemingly plausible explanation for this unusual pattern, according to the authors, is the adaptation of the diagnosis in patients with advanced OA disease. The sample size for this study was relatively small, and the authors acknowledge that there were only three depressed patients with KL 3 or higher at the time of the first follow-up, thus producing a particularly large confidence interval. In addition, it has been shown that the relationship between depressive symptoms and knee pain/functional deterioration is independent of the radiographic severity of $\mathrm{OA}^{28,29}$ A study by Veronese et al also found that the strength of the association between KOA and depressive symptoms decreased when radiographic/clinical KOA was used instead of selfreported KOA, and was not significant at the $\mathrm{P}<0.05$ level (adjusted OR: $1.32 ; 95 \% \mathrm{CI}$ : $0.99-1.78 ; \mathrm{P}=0.06$ ) ${ }^{19}$ These studies suggest that OA symptom burden, such as pain and physical functional limitations, is much more strongly associated with depressive symptoms than radiological grading.

\section{Depression as a Risk Factor for OA}

Recently, Wang et al developed a symptomatic KOA prediction model using secondary analysis of the China Health and Retirement Longitudinal Study data to identify individuals at risk of developing KOA. ${ }^{30}$ The authors concluded that previous KOA prediction models had ignored many potential predictors; therefore, this study included depressive symptoms variables in the model. They found that patients with mild or moderate to severe depression had a two- or three-times higher risk of developing KOA than those without depression. ${ }^{30}$ The risk model showed good discrimination with area under the receiver operating characteristic curve $(\mathrm{AUC})=0.719(95 \% \mathrm{CI}=0.700-0.737)$ and optimismcorrected $\mathrm{AUC}=0.712$ after bootstrap validation. Similar studies are rare; thus, there is a lack of robust data to support such a hypothesis. This seems logical, as the research on comorbid depression has often had the goal of improving OA management. In other words, the focus has been on depression treatment in patients with OA rather than OA prevention in patients with depression. In conclusion, it is inconclusive whether depression increases the risk of OA onset.

The association between depressive symptoms and OA symptom progression and structural progression has been documented in several longitudinal studies. Rathbun et al found that depressive symptoms had a nonlinear direct effect 
on the physical functioning of patients with KOA and an indirect effect mediated through pain severity. ${ }^{31}$ They also found that the persistence of depressive symptoms had a cumulative effect on knee pain. ${ }^{32}$ These studies suggest that depressive symptoms may lead to prolonged and increased OA pain, thus creating a vicious cycle between depression and OA pain. Using a group-based trajectory model to analyze the Osteoarthritis Initiative data, White et al found that baseline depressive symptoms were associated with functional decline trajectories. ${ }^{33}$ This is supported by a recent systematic review with moderate evidence supporting depression as a predictor of severe or progressive OA pain trajectory. ${ }^{34}$ Moreover, Jacobs et al used biomarker analysis to identify structural changes in KOA after two years of follow-up of non-obese patients, obese patients without depression, and obese patients with depressed mood. ${ }^{35}$ They found that obese patients with depressed mood had significantly worse pain and function at baseline and 2-year follow-up compared to the other two groups. In addition, an increased excretion of collagen terminal peptide markers was observed in the urine, thereby suggesting a more severe progression in cartilage degradation and bone reconstruction. ${ }^{35}$ Both obesity and depression have been separately identified as increasing the risk of systemic disease. In this study, the authors suggest that the increased cartilage degeneration and bone remodeling in obese patients with comorbid depression may be due to an increased systemic inflammatory burden compared to non-obese patients or obese patients without depression. This is an important finding, because very few previous studies have used biomarker approaches to assess the risk of disease progression in OA patients suffering from depression. These findings may suggest that depression is not only important for OA symptom progression, but may also affect the structural progression of OA.

However, some of the findings are inconsistent. A prospective study found no significant difference in the progression trajectory of OA structural deterioration based on radiology between patients with KOA with and without depression. ${ }^{36}$ Notably, the authors suggested the possibility of different conclusions if more sensitive methods had been used to assess structural OA progression. ${ }^{36}$ This may also be part of the reason for the inconsistent conclusions reached by Jacobs and Rathbun et $\mathrm{al}^{24,35}$ Furthermore, Riddle et al found that among patients with depression, the Western Ontario and McMaster Universities Osteoarthritis (WOMAC) pain scores and functional scores worsened at average rates of 0.6 and approximately 2 points per year, respectively. ${ }^{37}$ This suggests that some thresholds, such as baseline mental health and depressive symptom severity and duration, may need to be met for depression to have a clinically significant effect on OA symptoms. In addition, conflicting findings suggest that different depression subtypes are not consistently associated with OA. Rathbun et al found that patients with OA have different subtypes of depression that may have different effects on pain and disability. ${ }^{38}$ In a next exploratory study, López-Ruiz et al found that compared to control subjects, patients with OA were more likely to exhibit a temperamental self-image, dramatic speech, be submissive in relationships, be irritable, express resentment, feel blamed and ashamed, and exhibit physical weakness, fatigue, exaggerated physical symptoms, and health concerns. ${ }^{39}$ These multidimensional symptoms emphasize the heterogeneity of OA disorders, which may partially explain the contradictory results between some studies.

Overall, epidemiological studies on the relationship between OA and depression are rapidly increasing. However, this bidirectional relationship is still inconclusive. Future longitudinal studies should adequately discuss the mediating role of OA symptoms on OA depression. This is because not all patients with OA have the same symptoms, and some are even asymptomatic.

\section{Effects of Depression on OA Treatment Outcomes}

Over the past decade, there has been much discussion about the impact of depression on OA treatment outcomes, as evidenced by a recent systematic review. ${ }^{40}$ These studies adjusted for specific confounders and analyzed the relationship between patients' baseline depressive symptoms and OA prognosis. ${ }^{40}$ Most studies have focused on patients with knee and hip OA. Few have addressed those with hand, shoulder, or spine OA. Some but not all studies have concluded that preoperative depression is associated with poorer postoperative outcomes, such as greater postoperative pain, ${ }^{7,41-43}$ poor clinical and functional improvement, ${ }^{4-46}$ higher infection rates, ${ }^{6,47-49}$ and lower satisfaction. ${ }^{50}$ The recent systematic review also supports that depression has a negative impact on TJA outcomes. ${ }^{40}$ This systematic review, published in 2020, included 30 articles (16 retrospective and 14 prospective) comprehensively reviewing the relationship between depression and TJA in the last decade. ${ }^{40}$ In addition, several studies have examined the impact of depression on conservative treatment of $\mathrm{OA} .{ }^{51-53}$ 
Indeed, controversial results have emerged regarding the impact of depression on OA treatment outcomes. In 2014, Singh and Lewallen presented four key limitations of such studies. ${ }^{46}$ Subsequently, Bletterman analyzed in detail the reasons for the poor quality of prospective longitudinal studies. ${ }^{54}$ On this basis, these differences could be attributed partly to several factors.

First, the sensitivity of many early studies was limited by the failure to calculate trial sample sizes or the high rates of loss to follow-up. Vissers et al indicated that depression might have no effect on TKA outcomes. ${ }^{55}$ However, the following year, in a prospective multicenter study at three general hospitals in the Netherlands, Vissers et al calculated the sample size to reduce the risk of false negative results. ${ }^{42}$ The findings indicated that preoperative depressive symptoms were predictive of less change in the Knee injury and Osteoarthritis Outcome Scores and Hip disability and Osteoarthritis Outcome Scores 12 months after surgery. ${ }^{42}$

Second, patients with KOA, HOA, and lumbar spine OA exhibit different patterns of perioperative depression. ${ }^{56}$ Thus, the use of samples of patients with surgeries at different sites or primary and revision surgeries might have negatively affected the homogeneity of the cohorts. ${ }^{57}$ In addition, depression and OA symptoms can change over time. ${ }^{58}$ Some studies did not provide a comprehensive clinical assessment of patients over time, nor did they provide detailed sample characteristics. Future studies should collect information on changes in patients' conditions over a longitudinal course, as well as give information about the degree of depression or details of treatment.

Finally, results can be confounded by common issues, such as the use of various depression scales, inconsistent cutoff scores, and different study contexts, as well as the lack of clinical diagnoses and adequate adjustments for potential confounders (eg, use of antidepressants and analgesics) in observational studies. Therefore, the results and conclusions of the present study should be interpreted with caution. Improvements in the above areas are needed in future studies.

Overall, the current evidence suggests that most depressed patients can improve with TJA. However, a subset of patients will not improve. Future studies need to identify patient characteristics associated with depression non-resolution and need to identify the high risk group for persistent progression of depressive symptoms.

\section{Potential Mechanisms of OA and Comorbid Depression}

The wide range of risk factors associated with depression in OA suggests that there may be multiple potential comorbidity pathways, making it impossible to find a simple etiologic model. Furthermore, there is insufficient evidence to support depression as a risk factor for the development of OA. Therefore, in this paper, we elucidate the potential mechanisms by which OA increases the risk of depression from biological, sociological, and psychological perspectives through a biopsychosocial model. As shown in Figure 1. Biological and psychosocial factors are not parallel; they are interrelated.

\section{Biological Factors}

If the comorbidity of $\mathrm{OA}$ and depression is not coincidental, then there should be a biological link between the two disorders. In fact, several studies have confirmed the involvement of inflammation, neurotransmitters, the hypothalamicpituitary adrenal axis, and cortisol levels in the biological mechanisms of OA and depression. ${ }^{59-62}$

A growing body of literature has identified inflammatory mediators, such as interleukin-1 $\beta$, interleukin- 6 , and tumor necrosis factor- $\alpha$, in OA. ${ }^{60}$ It has been established that these peripheral cytokines can act on the central nervous system, which is distant from the joints. ${ }^{63}$ In a review, D'Mello and Swain detailed the main pathways of communication between the periphery and the brain, as well as the mechanisms by which inflammation plays a role in depression. ${ }^{64}$ Shimura et al observed that in patients with KOA, those with depression had higher serum interleukin-6 levels than those without. ${ }^{65}$ However, the sample size of this study was small. The cross-sectional study design also did not accurately reflect the causal relationship between inflammation and depression in patients with OA. Future prospective studies with large samples are needed to address these issues. In fact, any disease with a significant inflammatory component in the pathological mechanism is likely to induce depressive symptoms. ${ }^{66}$ OA that has an inflammatory component is no exception, which still needs to be verified by more studies.

Moreover, the comorbidity of OA and depression may be due, in part, to a common genetic etiology. Some studies have already addressed this problem by using large genetic data sets to identify risk variants and determine sites that 


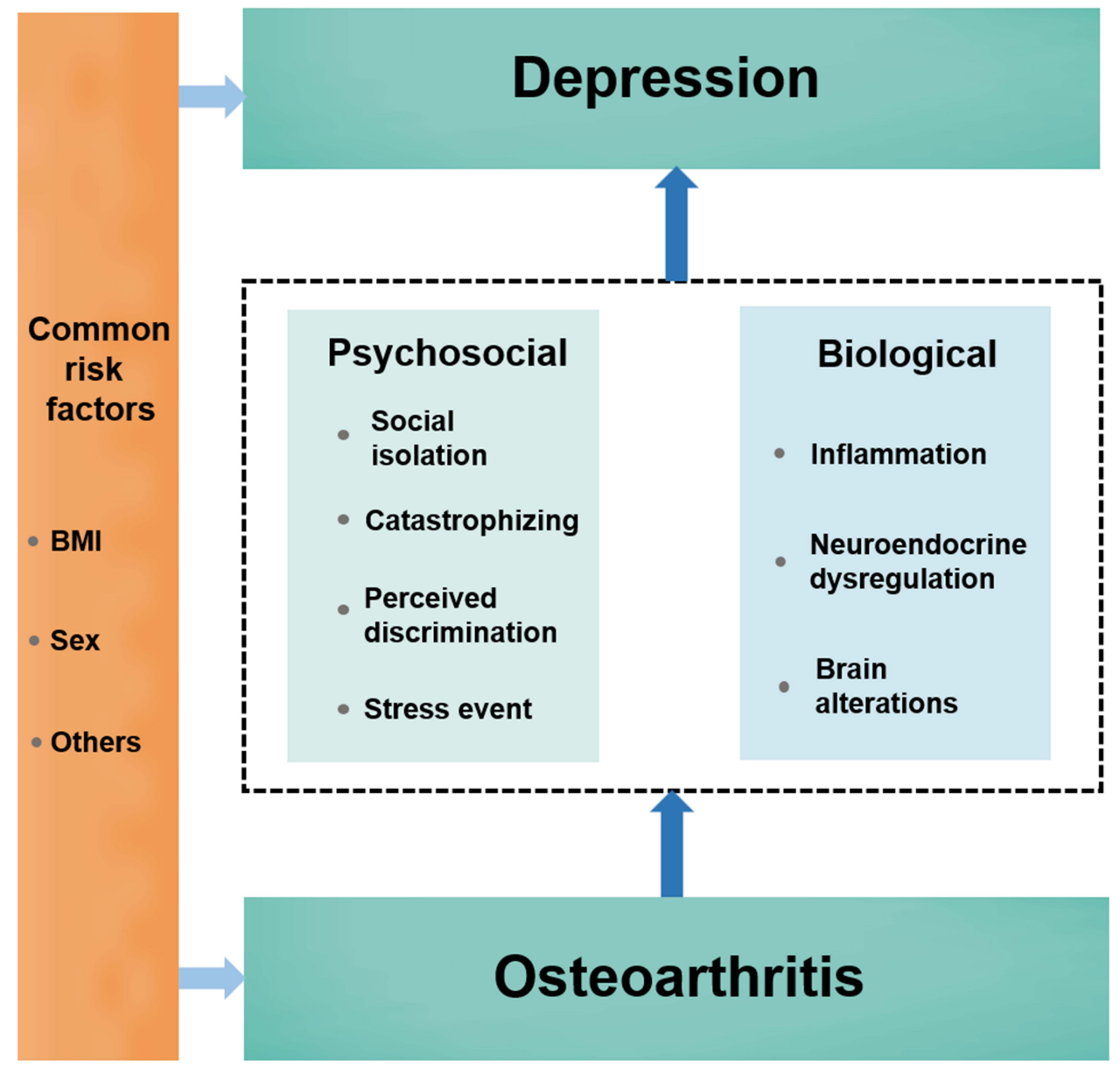

Figure I Potential mechanisms of comorbid depression in osteoarthritis.

Notes:A plausible explanation for the comorbidity of depression and OA may be influenced by a complex set of interacting factors that form a bidirectional relationship from psychosocial factors and biological mechanisms. There may also be common disease risk factors, which could go great lengths in explaining why depression and OA tend to coexist.

contribute to MDD risk. Recently, Barowsky et al used cross-disorder genome-wide genetic data analysis to examine the etiological relationship between OA and MDD. ${ }^{67}$ After a series of pleiotropic analyses, the findings suggest that $\mathrm{OA}$ and MDD share common genetic risk mechanisms, one of which centers on the neural response to the sensation of mechanical stimulus. ${ }^{67}$ This suggests that the two diseases may share a common genetic risk mechanism. In addition, Boer et al conducted a genome-wide association study meta-analysis across 826,690 individuals (177,517 with $\mathrm{OA}$ ) and found that $\mathrm{OA}$ shares genetic components with multiple traits, including depressive symptoms. ${ }^{68}$ More convincing evidence is needed in the future to analyze whether there is a genetic overlap between OA and depression.

Research findings based on neuroimaging techniques such as magnetic resonance imaging (MRI), provide a neural basis for the causal relationship between OA and depression. Pain is known to have a significant impact on brain function in areas such as cognition and emotion. ${ }^{69}$ MRI scans of the brains of people with OA have shown that people with OA have faster declines in hippocampal volume than people without OA and people with normal cognition, even after adjusting for factors such as age, gender, education, and APOE4 genotype ${ }^{70}$ Brain structural studies of people with depression have consistently found smaller hippocampal volumes in people with MDD compared to people without depression. ${ }^{70}$ It is clear that there is an association between the functional and structural changes in the specific brain regions involved in OA pain processing and depressive symptoms. In fact, few studies exist for the depression comorbid OA group, which makes it difficult to compare more clearly the changes in the brain of patients with OA and comorbid depression. 


\section{Psychosocial Factors}

The biopsychosocial model posits that health is influenced by biological, psychological, and social factors and affects the complex interplay of these factors. ${ }^{71}$ From the perspective of psychological research, patients with OA have more exposure to psychological and physical stressful events such as pain, physical limitations with other psychological stressors related to the diagnosis of OA than the general population. The link between stressful life events and depression has been found to be strong. ${ }^{72}$ Indeed, in addition to the fact that OA pain is itself a physiological stressor, many psychosocial variables have been shown to play a role in the relationship between OA and depression. Because there is no gold standard for the definition and classification of psychological constructs, different researchers have different ways of delineating them, and the amount of information available on psychosocial factors is enormous, we only review the relevant variables selectively. The psychosocial factors that have received more attention in OA include perceived discrimination, ${ }^{73}$ aesthetic discomfort, ${ }^{74}$ pain catastrophizing, ${ }^{75}$ social support, ${ }^{76}$ perceived isolation, ${ }^{77}$ social isolation, ${ }^{78}$ and neighborhood characteristics. ${ }^{79}$ Studies have been conducted to support the role of these psychological factors in the bidirectional relationship between OA and depression. Specifically, pain catastrophizing is often cited as a risk factor for the development of OA pain, ${ }^{80}$ making OA patients more likely to develop depressive symptoms by amplifying pain. In a cohort of older veterans with OA, McClendon et al found that the number of socially disadvantaged groups to which each participant belonged (ie, self-reported female gender, African American race, income $<\$ 20,000$, and/or unemployment due to disability) was significantly associated with higher perceived discrimination, pain, and depression, and that perceived discrimination significantly mediated the relationship between cumulative disadvantage and depressive symptoms, and pain severity. ${ }^{73}$

Using prospective data from the European Project on OSteoArthritis, Siviero et al found that people with KOA or HOA, regardless of whether they also had hand OA, had a 1.47-fold higher risk of social isolation than someone with no OA or only hand OA. ${ }^{78}$ This result is not surprising; pain and functional impairment have been shown to be risk factors for social isolation. Interestingly, this study also found that OA remained an independent predictor of social isolation in multivariate analyses after adjusting for pain and functional limitations. The authors interpreted this to mean that the fear of pain in OA patients was more important than OA pain itself. ${ }^{78}$ It is reasonable to speculate that the OA diagnosis itself may also cause psychological stress to the patient. In fact, qualitative studies have shown that most people have very little knowledge about $\mathrm{OA}^{40}$ The misconception that exercise causes joint damage and pain or that conservative treatments are ineffective undoubtedly increases the psychological stress of people with OA thereby promoting depression. A recent national study of Chinese patients with arthritis found that greater mobility impairment predicted an increased risk of depression. ${ }^{81}$ The authors concluded that it is unclear whether the ability to walk itself or the limitation of community participation due to walking difficulties leads to an increased risk of worsening depressive symptoms. This study did not distinguish between subtypes of arthritis; however, the findings are consistent with a study conducted by Parmelee et al in patients with OA. ${ }^{82}$ Parmelee et al found that the ability of older adults with KOA to engage in casual activities, such as engaging in hobbies or visiting friends or relatives, was associated with fewer depressive symptoms. ${ }^{82}$ It is reasonable to speculate that OA increases the risk of depression through psychosocial factors such as social isolation as a mediating variable, ie, OA directly or indirectly increases the risk of social isolation and subsequently induces depression. Of course, additional longitudinal studies are needed to assess the role of psychosocial factors in the relationship between $\mathrm{OA}$ and depression.

\section{Management of Depression in OA Screening for Depression}

The review of the OA literature indicated that the Hospital Anxiety and Depression Scale (HADS), CES-D, Beck Depression Inventory, Geriatric Depression Scale, and PHQ-9 have been commonly used to assess depression. The HADS has been shown to be valid and reliable in OA applications when the appropriate thresholds are used. ${ }^{83}$ The psychometric assessments of many scales are applicable to the general population or patients with chronic conditions; ${ }^{84}$ however, very limited data are available for individuals with OA. Thus, clinicians can refer to instruments that have been 
validated for disorders with symptoms similar to those in OA. Smarr and Keefer's review provided detailed recommendations for selecting a depression screening scale. ${ }^{85}$

There is no consensus on the depression screening procedures in clinical practice. At first glance, universal depression screening for patients with OA would appear to be beneficial; however, the decision should be evidence-based. Individual studies have investigated the possible impact of depression screening on clinical outcomes in samples of patients with OA. Mallen et al conducted a cluster RCT involving 45 UK general practices where GPs were randomized to routinely screen patients with OA for depression and anxiety or not. The primary outcome was current pain intensity reported by patients at post-visit and at 3-, 6-, and 12-month follow-up. Secondary outcomes included pain-related disability, anxiety, depression, and general health. Results from the multistage modeling showed that participants screened for depression had significantly higher mean pain scores than those in the control group at all follow-up time points. ${ }^{86}$ This researcher and colleagues used this data to report the cost-effectiveness of adding the above-mentioned measures of routine screening for anxiety and depression in older patients with OA. ${ }^{87}$ The results showed that this was not a cost-effective option. This cluster RCT filled an apparent gap in the literature; however, the results were surprising and disappointing. This is because most researchers advocate universal screening for depression in the OA population, thereby improving outcomes and reducing future health care burden and costs. To our knowledge, this is the first large primary care-based pragmatic trial to investigate the utility of this approach. The findings cast doubt on whether screening for anxiety and depression should be routinely performed in primary care in patients with OA. Unfortunately, because of the paucity of similar studies, a comparison of the studies was not possible. The value of screening for depression should not be dismissed because of the results of this study by Mallen et al. ${ }^{86}$ Subsequent research should validate the effectiveness and cost-effectiveness of different screening procedures in combination with treatment options.

\section{Nonpharmacological Treatment}

Given the high burden that depression poses in the OA population, there is an urgent need for advances in medical treatment. Research into this realm is ongoing. In 2019, the Osteoarthritis Research Society International (OARSI) updated its clinical guidelines for the nonsurgical management of knee, hip, and polyarticular OA. ${ }^{88}$ The effects of comorbidities, including depression, on treatment choices were acknowledged. Specifically, the guidelines suggest that clinicians choose mind-body exercise, cognitive behavioral therapy (CBT), and CBT combined with exercise as nonpharmacological intervention options for the subgroup of patients with OA with depression. The dominant therapy option is antidepressants, which are not a panacea, especially for older adults, who often suffer from multiple disorders. In addition, the number of studies evaluating the safety and efficacy of antidepressant classes in OA was small. ${ }^{89,90}$ Therefore, the present study has focused on psychotherapy and exercise therapy.

Most psychotherapy trials on OA populations have targeted surgical patients. These results have been summarized in systematic reviews and meta-analyses. A 2018 systematic review did not support the effectiveness of psychotherapy in TKA and total hip arthroplasty. ${ }^{91}$ However, most of the RCTs included in the systematic review were published before 2005, and their evidence was of low quality. ${ }^{91}$ A more recent meta-analysis included 40 studies. ${ }^{92}$ It concluded that perioperative interventions targeting psychological distress had a positive effect on postoperative pain, function, and QoL for patients who underwent TKA. ${ }^{92}$ Although not all of the evidence included in the meta-analysis was for psychotherapy, the results suggest that interventions targeting psychological distress in patients with TKA are very attractive directions for treatment. ${ }^{92}$

It should be noted that OA patients with depression may respond better to psychotherapy than those without psychological disorders. If the OA patients participating in the trial required little psychotherapy, there is little room for the intervention to be of value, which may obscure the true effect of psychotherapy on patients with OA comorbid depression. However, few studies have addressed this subgroup; thus, the clinical effects of psychotherapy are unclear. To address this limitation, Geng et al conducted an RCT. ${ }^{93}$ A total of 49 homogenous patients diagnosed with depression by a psychiatrist were enrolled in the trial. 25 patients in the intervention group received psychotherapy 4 times a week during hospitalization and every 2 weeks after discharge. The average number of psychotherapy sessions received by patients in the intervention group was $8.4 \pm 1.3$. It was found that at 6 months after TKA, the group receiving psychological interventions had significantly higher satisfaction than the control group receiving routine perioperative 
management ( $88 \%$ and $62.5 \%$, respectively). This finding supports the provision of comprehensive treatment, including psychotherapy, for depressed patients scheduled to undergo TKA. ${ }^{93}$ This study also confirms Bierke et al who found that patients' endogenous psychological characteristics had an impact on TKA satisfaction, with a particularly significant role of depression. ${ }^{50}$ The results of these two studies imply that the negative impact of depression on TKA satisfaction can be improved by psychotherapy.

In recent years, psychotherapy has no longer been limited to face-to-face meetings between physicians and patients. The telephone, internet, and mobile applications are increasingly being used. In a study by O'Moore et al, 69 patients with a combination of KOA and MDD were randomly assigned to internet-based cognitive behavioral therapy (iCBT) and conventional OA treatment groups. ${ }^{94}$ The participants in the iCBT group had fewer depressive symptoms, higher self-efficacy, less pain, and greater physical functioning at the completion of the intervention and at the 3-month followup. ${ }^{94}$ The results of this study are encouraging. They indicated that telemedicine may be attractive to patients who have difficulty attending in-person treatment, especially in the context of the coronavirus disease pandemic.

Exercise as an effective treatment for depression is also supported by the literature in patients with OA. A 2018 review of a mixed methods Cochrane study on the effects of exercise on the physical and mental health of patients with symptomatic OA of the lower extremities found moderate quality evidence that exercise can slightly alleviate depressive symptoms in individuals with OA. ${ }^{95}$ Although exercise has been shown to have a positive effect on physical and mental health improvement in OA, a question to consider is whether depression affects the response to exercise therapy in patients with OA. Legha et al used data from three large RCTs of exercise interventions in patients with KOA conducted in the United Kingdom, with the aim of investigating whether comorbidity was associated with primary outcomes based on WOMAC scores after KOA received exercise interventions. ${ }^{52}$ The study found that the presence of anxiety/depression was significantly associated with less pain and functional improvement at 6 months after the exercise intervention compared to the absence of anxiety/depression. This suggests that for people with OA depression comorbidity, a combination of other interventions (eg, pharmacotherapy and psychotherapy) is needed to compensate for the negative impact of depression on exercise therapy. Recently, an observational one-arm design study found similar levels of improvement in physical function, pain intensity, and QoL after 8 weeks of supervised exercise and educational interventions in individuals with $\mathrm{OA}$ and depression and those without depression. ${ }^{96}$ This finding suggests that supervised exercise can be used in concert with educational interventions.

In fact, de Rooij et al developed an individualized exercise program for KOA older adults with comorbidities as early as $2014 .{ }^{97}$ The authors concluded that disease interactions and their management require more complex and individualized care than simply the aggregate of separate guideline components. To develop an exercise program adapted to the comorbidities, the authors took five steps, which were to select comorbidities with a high prevalence of OA, conduct a literature search to identify limitations and contraindications to exercise therapy for each comorbidity, consult with experts in each comorbidity (who provided advice on the treatment of each comorbidity and how to incorporate principles of exercise therapy and training for the comorbidity into an exercise program for KOA), and conduct an exercise program in 11 patients to field test the protocol. Post-treatment evaluation of patient outcomes showed significant improvements in activity limitation and pain. This study design is not an RCT and therefore can only be considered to present an exploratory conceptual model of an exercise protocol for OA comorbidities. Future strong experimental data are needed to support that OA patients with comorbid depressive symptoms experience not only improved pain and physical function, but also improved mental health after exercise therapy.

For the first time, the latest OARSI guidelines include mind-body exercises, such as tai chi and yoga, as a core treatment option for patients with KOA. ${ }^{88}$ Currently, multimodal mind-body programs for OA patients with comorbid depression are being developed. ${ }^{98}$ The results of these studies are likely to change the current treatment paradigm. Furthermore, a recent network meta-analysis found that strengthening exercise, mixed exercise and control interventions were more beneficial than stretching for depressive symptoms. ${ }^{99}$ Strengthening exercises and mind-body exercises had comparable probability for optimal treatment of depressive symptoms. ${ }^{99}$ The authors concluded that health professionals can recommend strengthening exercises or mixed exercises as types of exercises to improve depressive symptoms in patients with KOA. ${ }^{99}$ A recent study also provides insight into designing exercise programs for people with depression. Stevens et al found that people's participation in sports groups can have benefits through the dual pathway of increased 
physical activity and decreased feelings of isolation. ${ }^{100}$ More research is needed in the future to determine the best exercise strategy for people with comorbid depression with OA.

\section{Conclusions and Future Directions}

In this review, up-to-date information has been gathered for analyzing the impact of depression on OA development and treatment response, elucidating potential mechanisms of comorbidity, reviewing the available evidence on depression screening, and exploring nonpharmacological treatments for depression appropriate for patients with OA. Much of what we know about depression in OA comes from epidemiological studies, such as prevalence, risk factors, and the bidirectional relationship between depression and OA. The incidence of depression varies considerably across the literature. However, the extant literature clearly indicates that patients with OA are more likely to have depressive symptoms as compared to the general population. Also, a large amount of this literature shows that patients with OA who suffer from depression tend to have worse clinical outcomes. The development of comorbid depression in OA involves multiple pathways, such as common biological and psychosocial factors. The safety and efficacy of many depression treatments based on underlying mechanisms have been demonstrated in patients with OA. Although some progress has been made in the understanding of the relationship between depression and OA, many questions remain unaddressed.

First, the relationship between depression and OA may be related to a complex set of factors, such as demographic factors (age, gender, race/ethnicity, economic conditions, education, employment), anthropometric traits (BMI, obesity), psychosocial factors (anxiety, pain catastrophizing, expectations, perceived discrimination, social support, social isolation, neighborhood characteristics), clinically relevant factors (pain, functional status, medical comorbidities, total number of troublesome joints), and other characteristics (fatigue, sleep). This means that disentangling the association between OA and depression and potential mediators remains an important avenue for future research. In addition, since psychological factors such as anxiety and pain catastrophizing often coexist with depression, future prospective studies are needed to comprehensively assess the relative contribution of each psychological factor to clinical outcomes in OA. The strategic next step would be to enhance our understanding of the mechanisms of this association with longitudinal studies with different types of OA patients, larger sample sizes, longer follow-up times, and utilizing more diverse statistical modeling approaches.

Second, the lack of focus on shared neurobiological mechanisms is a key limitation of the existing literature. Although there is a partial overlap between the changes in biochemical, molecular biological and neurobiological mechanisms observed in the two disorders separately, few studies have compared OA patients with comorbid depression to OA patients without depression. This is due in part to the fact that our understanding of the pathophysiology of depression alone and OA alone is extremely limited. Such studies have also received limited financial support. ${ }^{101}$ Considering the heterogeneity of OA and depression, it may be that only the elucidation of the biological mechanisms of MDD combined with OA disease will fundamentally change the current treatment situation.

From a clinical perspective, our first challenge is the timely screening and diagnosis of depression. It is very important to detect and provide timely treatment before depressive symptoms worsen further. To date, it is unclear when, where, and by whom depression screening is performed in patients with OA as well as what characteristics these patients meet. It is necessary to utilize appropriately designed, well-conducted prospective studies and RCTs in order to validate whether systematic depression screening leads to improved outcomes in patients with comorbid depression in OA. ${ }^{86}$ Furthermore, focused screening of those patients with OA who are at greatest risk for depression may not only reduce unnecessary psychological harm but also avoid the consumption of medical resources. Therefore, there is a clear need for future research to construct simple and easy to use clinical prediction models for depression. Also, the psychometric properties of depression screening tools should be tested among the OA population in the future, as this will assist in advancing the understanding that surrounds the selection of screening tools and optimal cutoff scores.

Available evidence supports psychological interventions and exercise therapy for patients with OA who suffer from depression, but these results need to be replicated and expanded. We found a lack of evaluation of the cost and effectiveness of other interventions, such as physical therapy, pharmacotherapy, and integrated care models, in this unique subgroup. Therefore, initiating studies to determine whether physiotherapy approaches, such as transcranial magnetic stimulation or electroconvulsive therapy for MDD and phonophoresis or transcutaneous electric nerve stimulation for pain relief, might help with depression in OA may provide valuable new insights. In addition, little is 
known about the challenges and facilitators in the treatment of OA patients with comorbid depression, and future exploratory qualitative studies with patients and healthcare professionals are needed to address this issue. It is noteworthy that most of the current studies have targeted KOA, and this evidence cannot necessarily be extrapolated to patients with $\mathrm{OA}$ in different joints. ${ }^{88}$ Thus, there is a need to include patients with various types of OA. Importantly, the target population for intervention studies should be OA patients who already have depressive symptoms or are at a high risk for depression. Finally, further exploration of the short- and long-term efficacy and influences of various treatment options, mechanisms of action of treatment, and dose-effects is needed in the future. In conclusion, more research is needed to determine the most effective strategies for treating depressive symptoms in patients with OA.

The relationship between depression and OA is a very interesting topic. Several systematic reviews could be conducted in the future, such as the effect of depression on treatment mechanisms, effect of depression on OA screening etc. High-quality research is needed to fill the various gaps in this area and to develop clinical practice guidelines applicable to patients with OA as soon as possible, specifically in the case of those with comorbid depression. Health practitioners need to recognize depression as an important comorbidity of OA; thus, they should comprehensively assess patients' mental health. ${ }^{88}$ In addition, interdisciplinary collaboration is needed to design and implement individualized treatment plans to improve patient QoL.

\section{Abbreviations}

OA, osteoarthritis; OR, odds ratio; CI, confidence interval; TKA, total knee arthroplasty; QoL, quality of life; HRQoL, Health-related quality of life; KOA, knee osteoarthritis; TJA, total joint arthroplasty; RA, rheumatoid arthritis; MDD, major depressive disorder; PHQ-9, Patient Health Questionnaire-9; BMI, body mass index; CES-D, Center for Epidemiological Studies-Depression; HOA, hip osteoarthritis; RCTs, randomized controlled trials; KL, KellgrenLawrence grade; AUC, area under the receiver operating characteristic curve; WOMAC, the Western Ontario and McMaster Universities Osteoarthritis; MRI, magnetic resonance imaging; HADS, Hospital Anxiety and Depression Scale; OARSI, Osteoarthritis Research Society International; CBT, cognitive behavioral therapy; iCBT, internet-based cognitive behavioral therapy.

\section{Disclosure}

The authors report no conflicts of interest in this work.

\section{References}

1. James SL, Abate D, Abate KH, et al. Global, regional, and national incidence, prevalence, and years lived with disability for 354 diseases and injuries for 195 countries and territories, 1990-2017: a systematic analysis for the Global Burden of Disease Study 2017. Lancet. 2018;392 (10159):1789-1858. doi:10.1016/S0140-6736(18)32279-7

2. Puig-Junoy J, Ruiz Zamora A. Socio-economic costs of osteoarthritis: a systematic review of cost-of-illness studies. Semin Arthritis Rheum. 2015;44(5):531-541. doi:10.1016/j.semarthrit.2014.10.012

3. Dziedzic KS, Allen KD. Challenges and controversies of complex interventions in osteoarthritis management: recognizing inappropriate and discordant care. Rheumatol Oxf Engl. 2018;57(suppl_4):iv88-iv98. doi:10.1093/rheumatology/key062

4. Stubbs B, Aluko Y, Myint PK, Smith TO. Prevalence of depressive symptoms and anxiety in osteoarthritis: a systematic review and meta-analysis. Age Ageing. 2016;45(2):228-235. doi:10.1093/ageing/afw001

5. Agustini B, Lotfaliany M, Woods RL, et al. Patterns of Association between Depressive Symptoms and Chronic Medical Morbidities in Older Adults. J Am Geriatr Soc. 2020;68(8):1834-1841. doi:10.1111/jgs.16468

6. Mollon B, Mahure SA, Ding DY, Zuckerman JD, Kwon YW. The influence of a history of clinical depression on peri-operative outcomes in elective total shoulder arthroplasty: a ten-year national analysis. Bone Jt J. 2016;98-B(6):818-824. doi:10.1302/0301-620X.98B6.37208

7. Pan X, Wang J, Lin Z, Dai W, Shi Z. Depression and Anxiety Are Risk Factors for Postoperative Pain-Related Symptoms and Complications in Patients Undergoing Primary Total Knee Arthroplasty in the United States. J Arthroplasty. 2019;34(10):2337-2346. doi:10.1016/j. arth.2019.05.035

8. Agarwal P, Sambamoorthi U. Healthcare Expenditures Associated with Depression Among Individuals with Osteoarthritis: post-Regression Linear Decomposition Approach. J Gen Intern Med. 2015;30(12):1803-1811. doi:10.1007/s11606-015-3393-4

9. Tsuji T, Nakata K, Vietri J, Jaffe DH. The added burden of depression in patients with osteoarthritis in Japan. Clin Outcomes Res. 2019;11:411-421. doi:10.2147/CEOR.S189610

10. Tan V, Jinks C, Chew-Graham C, Healey EL, Mallen C. The triple whammy anxiety depression and osteoarthritis in long-term conditions. $B M C$ Fam Pract. 2015;16(1):163. doi:10.1186/s12875-015-0346-2

11. Guglielmo D, Hootman JM, Boring MA, et al. Symptoms of Anxiety and Depression Among Adults with Arthritis — United States, 20152017. MMWR Morb Mortal Wkly Rep. 2018;67(39):1081-1087. doi:10.15585/mmwr.mm6739a2 
12. Scott JE, Mathias JL, Kneebone AC. Depression and anxiety after total joint replacement among older adults: a meta-analysis. Aging Ment Health. 2016;20(12):1243-1254. doi:10.1080/13607863.2015.1072801

13. Gandhi R, Zywiel MG, Mahomed NN, Perruccio AV. Depression and the Overall Burden of Painful Joints: an Examination among Individuals Undergoing Hip and Knee Replacement for Osteoarthritis. Arthritis. 2015;2015:1-6. doi:10.1155/2015/327161

14. Rosemann T, Backenstrass M, Joest K, Rosemann A, Szecsenyi J, Laux G. Predictors of depression in a sample of 1,021 primary care patients with osteoarthritis. Arthritis Rheum. 2007;57(3):415-422. doi:10.1002/art.22624

15. Pereira D, Severo M, Barros H, Branco J, Santos RA, Ramos E. The effect of depressive symptoms on the association between radiographic osteoarthritis and knee pain: a cross-sectional study. BMC Musculoskelet Disord. 2013;14(1):214. doi:10.1186/1471-2474-14-214

16. Zheng S, Tu L, Cicuttini F, et al. Depression in patients with knee osteoarthritis: risk factors and associations with joint symptoms. BMC Musculoskelet Disord. 2021;22(1):40. doi:10.1186/s12891-020-03875-1

17. Park H, Kim H, Lee Y. Knee osteoarthritis and its association with mental health and health-related quality of life: a nationwide cross-sectional study. Geriatr Gerontol Int. 2020;20(4):379-383. doi:10.1111/ggi.13879

18. Sale JEM, Gignac M, Hawker G. The relationship between disease symptoms, life events, coping and treatment, and depression among older adults with osteoarthritis. J Rheumatol. 2019;9.

19. Veronese N, Stubbs B, Solmi M, et al. Association between lower limb osteoarthritis and incidence of depressive symptoms: data from the osteoarthritis initiative. Age Ageing. 2017;46(3):470-476. doi:10.1093/ageing/afw216

20. Dell'Isola A, Pihl K, Turkiewicz A, et al. Risk of comorbidities following physician-diagnosed knee or hip osteoarthritis: a register-based cohort study. Arthritis Care Res. 2021:acr.24717. doi:10.1002/acr.24717

21. Li M, Nie Y, Zeng Y, et al. The trajectories of depression symptoms and comorbidity in knee osteoarthritis subjects. Clin Rheumatol. 2021. doi:10.1007/s10067-021-05847-9

22. Sugai K, Takeda-Imai F, Michikawa T, Nakamura T, Takebayashi T, Nishiwaki Y. Association Between Knee Pain, Impaired Function, and Development of Depressive Symptoms. J Am Geriatr Soc. 2018;66(3):570-576. doi:10.1111/jgs.15259

23. White DK, Neogi T, Zhang Y, Niu J, Katz PP. Association of Slow Gait Speed With Trajectories of Worsening Depressive Symptoms in Knee Osteoarthritis: an Observational Study: gait Speed and Depressive Symptoms in Knee OA. Arthritis Care Res. 2017;69(2):209-215. doi:10.1002/acr.22928

24. Rathbun AM, Shardell MD, Ryan AS, et al. Association between disease progression and depression onset in persons with radiographic knee osteoarthritis. Rheumatology. 2020;59(11):3390-3399. doi:10.1093/rheumatology/keaa141

25. IsHak WW, Wen RY, Naghdechi L, et al. Pain and Depression: a Systematic Review. Pain Depress. $2018 ; 26(6): 12$.

26. Fonseca-Rodrigues D, Rodrigues A, Martins T, et al. Correlation between pain severity and levels of anxiety and depression in osteoarthritis patients: a systematic review and meta-analysis. Rheumatology. 2021;61:53-75. doi:10.1093/rheumatology/keab512

27. Sayre EC, Esdaile JM, Kopec JA, et al. Specific manifestations of knee osteoarthritis predict depression and anxiety years in the future: Vancouver Longitudinal Study of Early Knee Osteoarthritis. BMC Musculoskelet Disord. 2020;21(1):467. doi:10.1186/s12891-020-03496-8

28. Alabajos-Cea A, Herrero-Manley L, Suso-Martí L, Alonso-Pérez-Barquero J, Viosca-Herrero E. Are Psychosocial Factors Determinant in the Pain and Social Participation of Patients with Early Knee Osteoarthritis? A Cross-Sectional Study. Int J Environ Res Public Health. 2021;18 (9):4575. doi:10.3390/ijerph18094575

29. Iijima H, Aoyama T, Fukutani N, et al. Psychological health is associated with knee pain and physical function in patients with knee osteoarthritis: an exploratory cross-sectional study. BMC Psychol. 2018;6(1):19. doi:10.1186/s40359-018-0234-3

30. Wang L, Lu H, Chen H, Jin S, Wang M, Shang S. Development of a model for predicting the 4-year risk of symptomatic knee osteoarthritis in China: a longitudinal cohort study. Arthritis Res Ther. 2021;23(1):65. doi:10.1186/s13075-021-02447-5

31. Rathbun AM, Shardell MD, Stuart EA, et al. Pain severity as a mediator of the association between depressive symptoms and physical performance in knee osteoarthritis. Osteoarthritis Cartilage. 2018;26(11):1453-1460. doi:10.1016/j.joca.2018.07.016

32. Rathbun AM, Stuart EA, Shardell M, Yau MS, Baumgarten M, Hochberg MC. Dynamic Effects of Depressive Symptoms on Osteoarthritis Knee Pain. Arthritis Care Res. 2018;70(1):80-88. doi:10.1002/acr.23239

33. White DK, Neogi T, Nguyen USDT, Niu J, Zhang Y. Trajectories of functional decline in knee osteoarthritis: the Osteoarthritis Initiative. Rheumatology. 2016;55(5):801-808. doi:10.1093/rheumatology/kev419

34. Previtali D, Andriolo L, Di Laura Frattura G, et al. Pain Trajectories in Knee Osteoarthritis-A Systematic Review and Best Evidence Synthesis on Pain Predictors. J Clin Med. 2020;9(9):2828. doi:10.3390/jcm9092828

35. Jacobs CA, Vranceanu AM, Thompson KL, Lattermann C. Rapid Progression of Knee Pain and Osteoarthritis Biomarkers Greatest for Patients with Combined Obesity and Depression: data from the Osteoarthritis Initiative. CARTILAGE. 2020;11(1):38-46. doi:10.1177/ 1947603518777577

36. Rathbun AM, Yau MS, Shardell M, Stuart EA, Hochberg MC. Depressive symptoms and structural disease progression in knee osteoarthritis: data from the Osteoarthritis Initiative. Clin Rheumatol. 2017;36(1):155-163. doi:10.1007/s10067-016-3495-3

37. Riddle DL, Kong X, Fitzgerald GK. Psychological health impact on 2-year changes in pain and function in persons with knee pain: data from the Osteoarthritis Initiative. Osteoarthritis Cartilage. 2011;19(9):1095-1101. doi:10.1016/j.joca.2011.06.003

38. Rathbun AM, Schuler MS, Stuart EA, et al. Depression Subtypes in Individuals With or at Risk for Symptomatic Knee Osteoarthritis. Arthritis Care Res. 2020;72(5):669-678. doi:10.1002/acr.23898

39. López-Ruiz M, Losilla JM, Monfort J, et al. Central sensitization in knee osteoarthritis and fibromyalgia: beyond depression and anxiety. PLoS One. 2019;14(12):e0225836. doi:10.1371/journal.pone.0225836

40. Vajapey SP, McKeon JF, Krueger CA, Spitzer AI. Outcomes of total joint arthroplasty in patients with depression: a systematic review. $J$ Clin Orthop Trauma. 2021;18:187-198. doi:10.1016/j.jcot.2021.04.028

41. Lewis GN, Rice DA, McNair PJ, Kluger M. Predictors of persistent pain after total knee arthroplasty: a systematic review and meta-analysis. $B r$ J Anaesth. 2015;114(4):551-561. doi:10.1093/bja/aeu441

42. Duivenvoorden T, Vissers MM, Verhaar JAN, et al. Anxiety and depressive symptoms before and after total hip and knee arthroplasty: a prospective multicentre study. Osteoarthritis Cartilage. 2013;21(12):1834-1840. doi:10.1016/j.joca.2013.08.022

43. Singh JA, Lewallen D. Predictors of pain and use of pain medications following primary Total Hip Arthroplasty (THA): 5,707 THAs at 2-years and 3,289 THAs at 5-years. BMC Musculoskelet Disord. 2010;11(1):90. doi:10.1186/1471-2474-11-90 
44. Hanusch BC, O’Connor DB, Ions P, Scott A, Gregg PJ. Effects of psychological distress and perceptions of illness on recovery from total knee replacement. Bone Jt J. 2014;96-B(2):210-216. doi:10.1302/0301-620X.96B2.31136

45. Kohring JM, Erickson JA, Anderson MB, Gililland JM, Peters CL, Pelt CE. Treated Versus Untreated Depression in Total Joint Arthroplasty Impacts Outcomes. J Arthroplasty. 2018;33(7):S81-S85. doi:10.1016/j.arth.2018.01.065

46. Singh JA, Lewallen DG. Depression in primary TKA and higher medical comorbidities in revision TKA are associated with suboptimal subjective improvement in knee function. BMC Musculoskelet Disord. 2014;15(1):127. doi:10.1186/1471-2474-15-127

47. Browne JA, Sandberg BF, D’Apuzzo MR, Novicoff WM. Depression Is Associated With Early Postoperative Outcomes Following Total Joint Arthroplasty: a Nationwide Database Study. J Arthroplasty. 2014;29(3):481-483. doi:10.1016/j.arth.2013.08.025

48. Ali AM, Loeffler MD, Aylin P, Bottle A. Factors Associated With 30-Day Readmission After Primary Total Hip Arthroplasty: analysis of 514 455 Procedures in the UK National Health Service. JAMA Surg. 2017;152(12):e173949. doi:10.1001/jamasurg.2017.3949

49. Yang Q, Lin Z, Yang S, Wang P, Chen R, Wang J. Incidence and Risk Factors of In-Hospital Prosthesis-Related Complications Following Total Knee Arthroplasty: a Retrospective Nationwide Inpatient Sample Database Study. Orthop Surg. 2021;13(5):1579-1586. doi:10.1111/ os. 13008

50. Bierke S, Häner M, Karpinski K, Hees T, Petersen W. Midterm Effect of Mental Factors on Pain, Function, and Patient Satisfaction 5 Years After Uncomplicated Total Knee Arthroplasty. J Arthroplasty. 2020;35(1):105-111. doi:10.1016/j.arth.2019.08.008

51. Chen YP, Huang YY, Wu Y, Kuo YJ, Lin CY. Depression negatively affects patient-reported knee functional outcome after intraarticular hyaluronic acid injection among geriatric patients with knee osteoarthritis. J Orthop Surg. 2019;14(1):387. doi:10.1186/s13018-019-1419-z

52. Legha A, Burke DL, Foster NE, et al. Do comorbidities predict pain and function in knee osteoarthritis following an exercise intervention, and do they moderate the effect of exercise? Analyses of data from three randomized controlled trials. Musculoskeletal Care. 2020;18(1):3-11. doi: $10.1002 / \mathrm{msc} .1425$

53. Uçkun AÇ. The Role of Pain Catastrophizing and Depression in the Outcomes of Physical Therapy in aProspective Osteoarthritis Cohort. Pain Physician. 2020;23(4):209-218. doi:10.36076/ppj.2020/23/209

54. Bletterman AN, de Geest-vrolijk ME, Vriezekolk JE. Preoperative psychosocial factors predicting patient's functional recovery after total knee or total hip arthroplasty: a systematic review. Clin Rehabil. 2018;32(4):512-525. doi:10.1177/0269215517730669

55. Vissers MM, Bussmann JB, Verhaar JAN, Busschbach JJV, Bierma-Zeinstra SMA, Reijman M. Psychological Factors Affecting the Outcome of Total Hip and Knee Arthroplasty: a Systematic Review. Semin Arthritis Rheum. 2012;41(4):576-588. doi:10.1016/j. semarthrit.2011.07.003

56. Power JD, Kudesia P, Nadeem A, et al. Patterns of Depressive Symptoms Before and After Surgery for Osteoarthritis: a Descriptive Study. $A C R$ Open Rheumatol. 2019;1(4):203-212. doi:10.1002/acr2.1031

57. Pinto PR, McIntyre T, Araújo-Soares V, Costa P, Ferrero R, Almeida A. A comparison of predictors and intensity of acute postsurgical pain in patients undergoing total hip and knee arthroplasty. J Pain Res. 2017;10:1087-1098. doi:10.2147/JPR.S126467

58. Visser MA, Howard KJ, Ellis HB. The Influence of Major Depressive Disorder at Both the Preoperative and Postoperative Evaluations for Total Knee Arthroplasty Outcomes. Pain Med. 2019;20(4):826-833. doi:10.1093/pm/pny107

59. Villafañe JH, Pedersini P, Bertozzi L, et al. Exploring the relationship between chronic pain and cortisol levels in subjects with osteoarthritis: results from a systematic review of the literature. Osteoarthritis Cartilage. 2020;28(5):572-580. doi:10.1016/j.joca.2020.02.836

60. Chow YY, Chin KY. The Role of Inflammation in the Pathogenesis of Osteoarthritis. Mediators Inflamm. 2020;2020:1-19. doi:10.1155/2020/ 8293921

61. Malhi GS, Mann JJ. Depression. The Lancet. 2018;392(10161):2299-2312. doi:10.1016/S0140-6736(18)31948-2

62. Dimmek DJ, Korallus C, Buyny S, et al. Brain-Derived Neurotrophic Factor and Immune Cells in Osteoarthritis, Chronic Low Back Pain, and Chronic Widespread Pain Patients: association with Anxiety and Depression. Medicina. 2021;57(4):327. doi:10.3390/medicina57040327

63. Berenbaum F, Meng QJ. The brain-joint axis in osteoarthritis: nerves, circadian clocks and beyond. Nat Rev Rheumatol. 2016;12(9):508-516. doi:10.1038/nrrheum.2016.93

64. D'Mello C, Swain MG. 2016. Immune-to-Brain Communication Pathways in Inflammation-Associated Sickness and Depression. In: Dantzer R, Capuron L, editors. Inflammation-Associated Depression: Evidence, Mechanisms and Implications. 31. 73-94. doi:10.1007/7854_2016_37

65. Shimura Y, Kurosawa H, Tsuchiya M, et al. Serum interleukin 6 levels are associated with depressive state of the patients with knee osteoarthritis irrespective of disease severity. Clin Rheumatol. 2017;36(12):2781-2787. doi:10.1007/s10067-017-3826-z

66. Gold SM, Köhler-Forsberg O, Moss-Morris R, et al. Comorbid depression in medical diseases. Nat Rev Dis Primer. 2020;6(1):69. doi:10.1038/ s41572-020-0200-2

67. Barowsky S, Jung JY, Nesbit N, et al. Cross-Disorder Genomics Data Analysis Elucidates a Shared Genetic Basis Between Major Depression and Osteoarthritis Pain. Front Genet. 2021;12:687687. doi:10.3389/fgene.2021.687687

68. Boer CG, Hatzikotoulas K, Southam L, et al. Deciphering osteoarthritis genetics across 826,690 individuals from 9 populations. Cell. $2021 ; 184$ (18):4784-4818.e17. doi:10.1016/j.cell.2021.07.038

69. Mutso AA, Radzicki D, Baliki MN, et al. Abnormalities in Hippocampal Functioning with Persistent Pain. J Neurosci. 2012;32(17):5747-5756. doi:10.1523/JNEUROSCI.0587-12.2012

70. Li X, Tong Q, Gao J, Liu C. Alzheimer's Disease Neuroimaging Initiative, Liu Y. Osteoarthritis Was Associated With a Faster Decline in Hippocampal Volumes in Cognitively Normal Older People. Front Aging Neurosci. 2020;12:190. doi:10.3389/fnagi.2020.00190

71. Keefe FJ, Smith SJ, Buffington ALH, Gibson J, Studts JL, Caldwell DS. Recent advances and future directions in the biopsychosocial assessment and treatment of arthritis. J Consult Clin Psychol. 2002;70(3):640-655. doi:10.1037/0022-006X.70.3.640

72. Hammen C. Risk Factors for Depression: an Autobiographical Review. Annu Rev Clin Psychol. 2018;14(1):1-28. doi:10.1146/annurev-clinpsy $-050817-084811$

73. McClendon J, Essien UR, Youk A, et al. Cumulative Disadvantage and Disparities in Depression and Pain Among Veterans With Osteoarthritis: the Role of Perceived Discrimination. Arthritis Care Res. 2021;73(1):11-17. doi:10.1002/acr.24481

74. Hodkinson B, Maheu E, Michon M, Carrat F, Berenbaum F. Assessment and determinants of aesthetic discomfort in hand osteoarthritis. Ann Rheum Dis. 2012;71(1):45-49. doi:10.1136/ard.2011.153965 
75. Gudmundsson P, Nakonezny PA, Lin J, Owhonda R, Richard H, Wells J. Functional improvement in hip pathology is related to improvement in anxiety, depression, and pain catastrophizing: an intricate link between physical and mental well-being. BMC Musculoskelet Disord. 2021;22 (1):133. doi:10.1186/s12891-021-04001-5

76. Lee C-T, Yeh C-J, Lee M-C, et al. Social support and mobility limitation as modifiable predictors of improvement in depressive symptoms in the elderly: results of a national longitudinal study. Arch Gerontol Geriatr. 2012;55(3):530-538. doi:10.1016/j.archger.2012.03.002

77. Santini ZI, Jose PE, York Cornwell E, et al. Social disconnectedness, perceived isolation, and symptoms of depression and anxiety among older Americans (NSHAP): a longitudinal mediation analysis. Lancet Public Health. 2020;5(1):e62-e70. doi:10.1016/S2468-2667(19)30230-0

78. Siviero P, Veronese N, Smith T, et al. Association Between Osteoarthritis and Social Isolation: data From the EPOSA Study. J Am Geriatr Soc. 2020;68(1):87-95. doi:10.1111/jgs.16159

79. Kowitt SD, Aiello AE, Callahan LF, et al. How Are Neighborhood Characteristics Associated With Mental and Physical Functioning Among Older Adults With Radiographic Knee Osteoarthritis? Arthritis Care Res. 2021;73(3):308-317. doi:10.1002/acr.24125

80. Fu K, Metcalf B, Bennell KL, et al. The association between psychological factors and pain exacerbations in hip osteoarthritis. Rheumatology. 2021;60(3):1291-1299. doi:10.1093/rheumatology/keaa494

81. Yang R, Wang J, Wang H, Tracy EL, Tracy CT. A cross-lagged model of depressive symptoms and mobility disability among middle-aged and older Chinese adults with arthritis. Geriatr Gerontol Int. 2020;20(10):873-877. doi:10.1111/ggi.13993

82. Parmelee PA, Tighe CA, Dautovich ND. Sleep Disturbance in Osteoarthritis: linkages With Pain, Disability, and Depressive Symptoms: osteoarthritis and Problems With Sleep Disturbance. Arthritis Care Res. 2015;67(3):358-365. doi:10.1002/acr.22459

83. Wong L, Yiu R, Chiu C, et al. Prevalence of Psychiatric Morbidity in Chinese Subjects with Knee Osteoarthritis in a Hong Kong. Orthopaedic Clinic. 2015;25:9.

84. Michela B, Cataldi F, Carlucci L, Padulo C, Fairfield B. Assessment of late-life depression via self-report measures: a review. Clin Interv Aging. 2018;13:2021-2044. doi:10.2147/CIA.S178943

85. Smarr KL, Keefer AL. Measures of Depression and Depressive Symptoms. Arthritis Care Res. 2020;72(S10):608-629. doi:10.1002/acr.24191

86. Mallen CD, Nicholl BI, Lewis M, et al. The effects of implementing a point-of-care electronic template to prompt routine anxiety and depression screening in patients consulting for osteoarthritis (the Primary Care Osteoarthritis Trial): a cluster randomised trial in primary care. PLOS Med. 2017;14(4):e1002273. doi:10.1371/journal.pmed.1002273

87. Kigozi J, Jowett S, Nicholl BI, et al. Cost-Utility Analysis of Routine Anxiety and Depression Screening in Patients Consulting for Osteoarthritis: results From a Clinical, Randomized Controlled Trial. Arthritis Care Res. 2018;70(12):1787-1794. doi:10.1002/acr.23568

88. Bannuru RR, Osani MC, Vaysbrot EE, et al. OARSI guidelines for the non-surgical management of knee, hip, and polyarticular osteoarthritis. Osteoarthritis Cartilage. 2019;27(11):1578-1589. doi:10.1016/j.joca.2019.06.011

89. van den Driest JJ, Schiphof D, de Wilde M, Bindels PJE, van der Lei J, Bierma-Zeinstra SMA. Antidepressant and anticonvulsant prescription rates in patients with osteoarthritis: a population-based cohort study. Rheumatology. 2021;60(5):2206-2216. doi:10.1093/rheumatology/keaa544

90. Ferreira GE, McLachlan AJ, Lin CWC, et al. Efficacy and safety of antidepressants for the treatment of back pain and osteoarthritis: systematic review and meta-analysis. BMJ. 2021:m4825. doi:10.1136/bmj.m4825

91. Bay S, Kuster L, McLean N, Byrnes M, Kuster MS. A systematic review of psychological interventions in total hip and knee arthroplasty. BMC Musculoskelet Disord. 2018;19(1):201. doi:10.1186/s12891-018-2121-8

92. Sorel JC, Overvliet GM, Gademan MGJ, den Haan C, Honig A, Poolman RW. The influence of perioperative interventions targeting psychological distress on clinical outcome after total knee arthroplasty. Rheumatol Int. 2020;40(12):1961-1986. doi:10.1007/s00296-02004644-y

93. Geng X, Wang X, Zhou G, et al. A Randomized Controlled Trial of Psychological Intervention to Improve Satisfaction for Patients with Depression Undergoing TKA: a 2-Year Follow-up. J Bone Jt Surg. 2021;103(7):567-574. doi:10.2106/JBJS.20.00169

94. O'moore KA, Newby JM, Andrews G, et al. Internet Cognitive-Behavioral Therapy for Depression in Older Adults With Knee Osteoarthritis: a Randomized Controlled Trial. Arthritis Care Res. 2018;70(1):61-70. doi:10.1002/acr.23257

95. Hurley M, Dickson K, Hallett R, et al. Exercise interventions and patient beliefs for people with hip, knee or hip and knee osteoarthritis: a mixed methods review. Cochrane Database Syst Rev. 2018. doi:10.1002/14651858.CD010842.pub2

96. Pihl K, Roos EM, Taylor RS, Grønne DT, Skou ST. Associations between comorbidities and immediate and one-year outcomes following supervised exercise therapy and patient education - a cohort study of 24,513 individuals with knee or hip osteoarthritis. Osteoarthritis Cartilage. 2021;29(1):39-49. doi:10.1016/j.joca.2020.11.001

97. de Rooij M, van der Leeden M, Avezaat E, et al. Development of comorbidity-adapted exercise protocols for patients with knee osteoarthritis. Clin Interv Aging. 2014:829. doi:10.2147/CIA.S55705

98. Jacobs CA, Mace RA, Greenberg J, et al. Development of a mind body program for obese knee osteoarthritis patients with comorbid depression. Contemp Clin Trials Commun. 2021;21:100720. doi:10.1016/j.conctc.2021.100720

99. Hall M, Dobson F, Van Ginckel A, et al. Comparative effectiveness of exercise programs for psychological well-being in knee osteoarthritis: a systematic review and network meta-analysis. Semin Arthritis Rheum. 2021;51(5):1023-1032. doi:10.1016/j.semarthrit.2021.07.007

100. Stevens M, Lieschke J, Cruwys T, Cárdenas D, Platow MJ, Reynolds KJ. Better together: how group-based physical activity protects against depression. Soc Sci Med. 2021;286:114337. doi:10.1016/j.socscimed.2021.114337

101. Nemeroff CB. The State of Our Understanding of the Pathophysiology and Optimal Treatment of Depression: glass Half Full or Half Empty? Am J Psychiatry. 2020;177(8):671-685. doi:10.1176/appi.ajp.2020.20060845 


\section{Publish your work in this journal}

Neuropsychiatric Disease and Treatment is an international, peer-reviewed journal of clinical therapeutics and pharmacology focusing on concise rapid reporting of clinical or pre-clinical studies on a range of neuropsychiatric and neurological disorders. This journal is indexed on PubMed Central, the 'PsycINFO' database and CAS, and is the official journal of The International Neuropsychiatric Association (INA). The manuscript management system is completely online and includes a very quick and fair peer-review system, which is all easy to use. Visit http://www.dovepress.com/testimonials.php to read real quotes from published authors. 